\title{
OTIMIZAÇÃO DE VOLUMES DE RESERVATÓRIOS DE ÁGUA DA CHUVA PARA USO PECUÁRIO
}

\section{OPTIMIZATION OF RAINWATER RESERVOIRS VOLUMES FOR LIVESTOCK USE}

Vinícius Velanes Giffoni

Mestrando do Programa de Pós-Graduação em Engenharia Civil e Engenharia Ambiental da Universidade Estadual de Feira de Santana. (vvelanes@hotmail.com)

\section{Eduardo Cohim}

Doutor Professor Adjunto do Departamento de Tecnologia da Universidade Estadual de Feira de Santana.(edcohim@gmail.com)

\section{Resumo}

A captação da água da chuva é uma antiga prática que tem como objetivo suprir as necessidades de água para irrigação, higiene pessoal e o consumo humano ou animal. O objetivo deste trabalho foi desenvolver um método de otimização econômica de sistemas de captação de água da chuva. Para o cálculo do volume, foram utilizados dados diários de precipitação, por um período de 20 anos, coletados em estações meteorológicas na região de Feira de Santana-BA. Foram analisados potenciais de atendimento variando de $65 \%$ a $95 \%$. Os resultados demonstraram que, para um potencial de atendimento adotado e uma dada demanda, existe uma infinidade de combinações entre área de captação e volume de reservatório, configurando uma indeterminação matemática, entretanto, há uma combinação que apresenta um custo mínimo. O método aplicado para as condições de Feira de Santana-BA resultou, para o sistema de aproveitamento ótimo, em um potencial de atendimento de $95 \%$ e demanda de $250 \mathrm{~L} /$ dia, numa área de captação de $208 \mathrm{~m}^{2}$ e volume de cisterna de $20.900 \mathrm{~L}$.

Palavras-Chave: Dimensionamento de reservatórios; caprinocultura; cisterna.

\section{Abstract}

Rainwater harvesting is an ancient practice that aims to meet the needs of water for irrigation, personal hygiene and human or animal consumption. The objective of this work was to develop a method of economical optimization of rainwater catchment systems. Volume calculation was based on daily rainfall data for a period of 20 years collected from meteorological stations in the Feira de Santana region. Service potentials ranging from $65 \%$ to $95 \%$ were analyzed. The results showed that, for a given service potential and a given demand, there is a multitude of combinations between catchment area and reservoir volume, thus constituting a mathematical indetermination, however, there is a combination that has a minimum cost. The method applied to the conditions of Feira de Santana-BA resulted in the optimum utilization system for a $95 \%$ service potential and $250 \mathrm{~L} /$ day demand, a $208 \mathrm{~m}^{2}$ catchment area and 20,900 L tank volume.

Keywords: Sizing of reservoirs; goat farming; cistern.

\section{INTRODUÇÃO E FUNDAMENTAÇÃO TEÓRICA}

A região semiárida brasileira é considerada uma das mais chuvosas do planeta, com precipitação média anual por volta de $750 \mathrm{~mm}$ (MONTENEGRO; MONTENEGRO, 2012). Por outro lado, a evapotranspiração, oriunda da ação do clima (AZEVÊDO et al., 2017) atinge uma média anual de $2500 \mathrm{~mm}$, o que acarreta elevados deficit hídricos que limitam a produtividade agrícola e pecuária da região. Além da distribuição irregular da precipitação no tempo, a região é caracterizada por solos rasos, rios intermitentes devido a solos pouco profundos e com baixa capacidade de infiltração, além de recursos hídricos subterrâneos escassos (ELLIOTT et al., 2014).

Segundo Cirilo (2008), a predominância de cursos d'água temporários aliados a secas prolongadas dificulta a gestão dos recursos 
hídricos na região, assim como a implementação de políticas públicas de combate à seca. Montenegro e Montenegro (2012) afirmam que esse quadro de incertezas provoca uma instabilidade na tomada de decisão de políticas de recursos hídricos e de desenvolvimento agropecuário, exigindo medidas de gestão que contribuam para o atendimento da demanda da população de forma contínua.

Se, por um lado, muito foi tentado no combate à seca através de obras emergenciais, sem cunho ambiental e com baixa durabilidade, por outro, o conceito de convivência com o semiárido vem modificando o modo com que cientistas, pesquisadores e o governo procuram soluções para a problemática da seca, adequando a demanda da população aos recursos oferecidos pelo meio ambiente, através de experiências de difusão de tecnologias apropriadas e de combate à seca e à desertificação (GUALDANI; FERNÁNDEZ; GUILLÉN, 2015).

Entre as principais tecnologias de convivência com o semiárido adotadas pelos programas governamentais, destacam-se aquelas que priorizam a agricultura e pecuária através da captação e armazenamento de água da chuva, fazendo uso de trincheiras, barragens subterrâneas e principalmente reservatórios. Os reservatórios de captação de água da chuva são uma prática de manejo hídrico sustentável, com um baixo impacto ambiental e vêm sendo adotados como medida alternativa para suprir a demanda de água em todo o mundo. Entre as principais necessidades atendidas por este método, estão as demandas domésticas e as de irrigação e pecuária no meio rural (CAMPISANO et al., 2017).

Embora os estudos sobre 0 dimensionamento de reservatórios de coleta de águas de chuva para uso doméstico sejam bastante numerosos (LONDRA et al., 2015), há uma falta de pesquisas relacionadas ao aproveitamento de água da chuva para o abastecimento animal. Entre as atividades produtivas no semiárido nordestino, destaca-se a caprinocultura, que é uma eficaz alternativa econômica para a região. Isto se justifica pela independência das cabras por grandes áreas de pastagens, além da sua baixa perda de água, o que torna esses animais resistentes à alta variação de temperaturas e ao estresse hídrico típico daquela região, diferentemente de outras espécies, como bovinos. (KALIBER; KOLUMAN;
SILANIKOVE, 2017; MAIA et al., 2018). Segundo dados do IBGE (2016), a região Nordeste abriga $93,0 \%$ do rebanho brasileiro de caprinos, o que equivale a 9,1 milhões de cabeças. O estado da Bahia, por sua vez, é responsável por $28,0 \%$ de todo o rebanho do Nordeste, equivalente a 2,5 milhões de cabeças. De acordo com Moraes Neto et al. (2003), a cultura de caprinos e ovinos representa uma boa alternativa de trabalho e renda, visto a produção de alimentos de alto teor proteico (leite, carne e vísceras), bem como de pele de excelente qualidade, além da adaptabilidade dos animais aos ecossistemas locais.

Entretanto, o custo para o abastecimento de água desses rebanhos torna a criação de animais uma prática inviável para muitos pequenos agricultores rurais (FRANÇA; HOLANDA JUNIOR; SOUSA NETO, 2011). Tal realidade caracteriza a água como um fator limitante para as atividades de produção, o que justifica a intensificação dos estudos sobre a disponibilidade de água, em quantidade e qualidade, e formas otimizadas de captação e reservação deste bem natural (PALHARES; GUIDONI, 2012). Assim, o objetivo deste trabalho é apresentar um método de dimensionamento e otimização de sistemas de captação de água da chuva, levando em consideração o custo de implantação dos sistemas. O uso desta metodologia deve possibilitar ao produtor do semiárido, além da definição do sistema de captação com o menor custo de implantação, optar pelo melhor sistema em função de sua disponibilidade de recursos, bem como limitar a aplicação do sistema quanto às dimensões de área de captação e ao volume da cisterna.

\section{METODOLOGIA}

Feira de Santana está localizada no centro norte baiano, numa planície entre o recôncavo e a região semiárida da Bahia. Seu regime de chuvas é bem definido, em que o quadrimestre de abril a julho concentra $43 \%$ do volume precipitado anualmente. Os dados de precipitação foram extraídos da plataforma do Instituto Nacional de Meteorologia (INMET, 2018), na estação de código 83221, no período de 01/01/1998 a $31 / 12 / 2017$, totalizando 20 anos de dados de precipitação. A demanda utilizada nos cálculos foi baseada no consumo médio diário de uma cabra de $5 \mathrm{~L} /$ dia. Todos os cenários foram simulados para um rebanho de 50 cabeças, totalizando 
250L/dia. A precipitação média anual para o médias mensais estão apresentadas na Figura 1. período estudado é de $664,2 \mathrm{~mm}$, enquanto as

Figura 1: Hietograma mensal de Feira de Santana no período 1998-2017

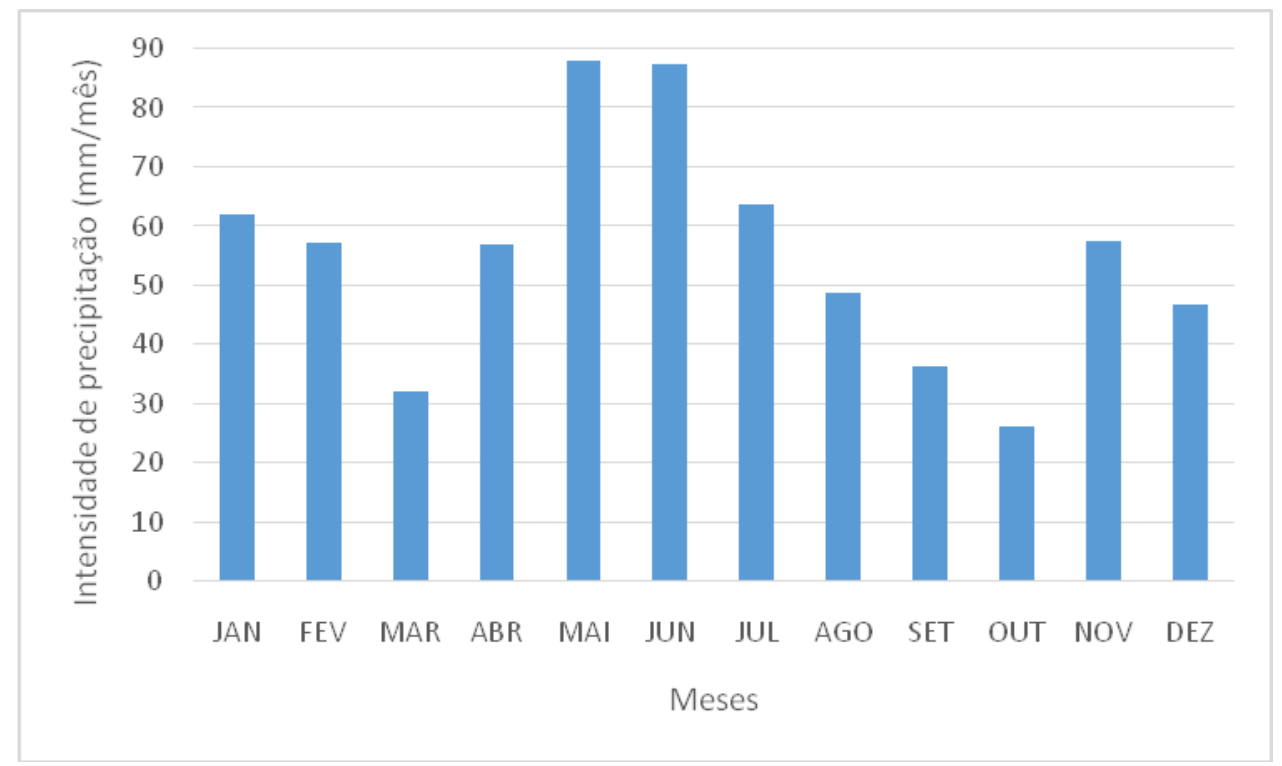

Fonte: Os autores, 2019.

O modelo utilizado foi desenvolvido por Jenkis e Pearson (1978) e aprimorado por Latham (1983), e está baseado no balanço seriado do comportamento do sistema com um intervalo diário (FEWKES, 2000), mostrado nas Equações 1 a 4.

As equações do modelo são apresentadas abaixo.

$Q_{[\mathrm{r}]}=P_{[\mathrm{s}]} x A x C$

$Y_{(t)}=\min \left\{\begin{array}{c}D_{[(t)} \\ V_{[(t-1)}+\theta Q_{[(t)}\end{array}\right\}$

$V_{(t)}=\min \left\{\begin{array}{c}\left(V_{(t-1)}+Q_{[(t)}-\theta Y_{(t)}\right)-(1-\theta) Y_{(t)} \\ R-(1-\theta) Y_{(t)}\end{array}\right\}(3)$

Sendo que: $\theta$ é um parâmetro adimensional que varia entre 0 e 1 . A utilização do coeficiente de $\theta=0$ descreve a liberação de água para 0 atendimento da demanda após o vertimento; já $\theta$ $=1$ corresponde ao comportamento do reservatório onde a demanda é atendida antes do vertimento. $O$ valor de $\theta$ adotado foi de 0,6 , contemplando uma situação intermediária entre esses extremos, sugerido por Fewkes (2000). O parâmetro $Q_{(t)}$ representa o volume escoado ao reservatório em função da precipitação, em L; a precipitação $P_{(t)}$, em $\mathrm{mm}$; a área de captação $A$, em $\mathrm{m}^{2} ; \mathrm{e}$ o coeficiente de escoamento $\mathrm{C}$, adimensional. Já $D_{\{(t)}$ é a demanda no período, em $L ; V_{(t)}$ é o volume de água armazenado naquele período, em L; e $Y_{(\mathrm{g})}$ é o volume de água utilizado para $\circ$ atendimento da demanda, no mesmo período, em L.

Para verificação do percentual de atendimento à demanda solicitada de água de chuva, foi adotada a seguinte formulação:

$$
P A(\%)=\frac{\sum Y_{(0)}}{\sum D} \times 100
$$

Em que $\sum Y_{(i))}$ corresponde ao somatório, durante o tempo avaliado, do volume de água liberado para o atendimento da demanda, e $\Sigma D$ é o somatório da demanda durante o período estudado.

Entre os critérios de análise, em função da variedade de parâmetros utilizados neste método, foi necessária a criação de um parâmetro normalizado adimensional com o intuito de generalizar as análises em função de outras variáveis, como volume ou custo. O parâmetro calculado foi o $F_{D}$ (Fração da Demanda), utilizado por Campisano e Modica (2012), quando determinaram volumes de reservatórios na Sicília (Itália). Este parâmetro expressa a relação entre a demanda anual (D), área de captação (A) e precipitação anual média (FEWKES, 2000). 
Abaixo, a Equação 5 representa o parâmetro citado.

$$
\mathrm{F}_{\mathrm{D}}=\frac{\mathrm{D}}{\mathrm{A} * \mathrm{P}}
$$

Os volumes de reservatório e suas respectivas áreas de captação foram calculados, considerando um percentual de garantia de atendimento à demanda de $65 \%, 75 \%, 85 \%$ e $95 \%$, pois a garantia de atendimento pode variar de acordo com a disponibilidade de outras fontes para o abastecimento de água.

O sistema de captação de água da chuva adotado foi o tipo calçadão, por ser uma tecnologia social de baixo custo e amplamente difundida no semiárido brasileiro. Esse sistema conta com cisternas tipo placas, produzidas com concreto no próprio local, através de mão de obra local. A área de captação ideal foi considerada, também, a partir de placas de concreto. A Figura 2 apresenta um esquema, em planta perfil, do sistema Área de Captação x Cisterna.

Figura 2: Desenho esquemático do sistema tipo calçadão (Planta baixa e Corte)

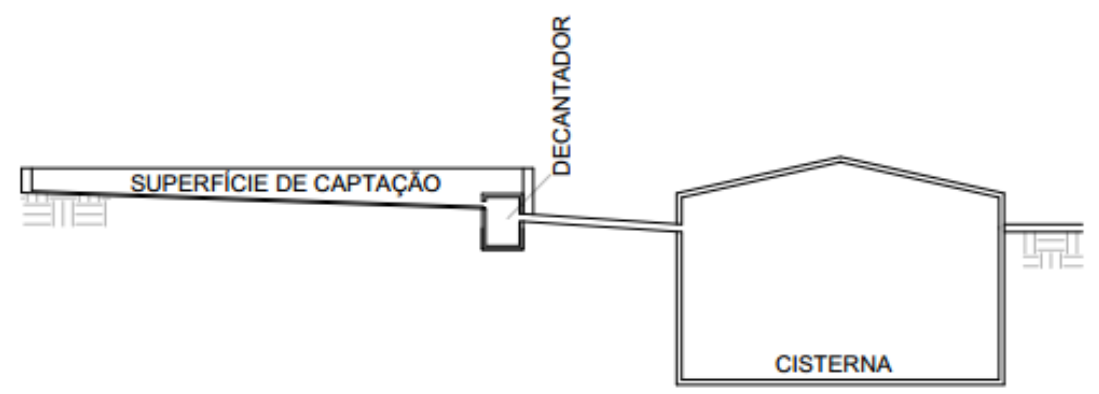

CORTE A-A

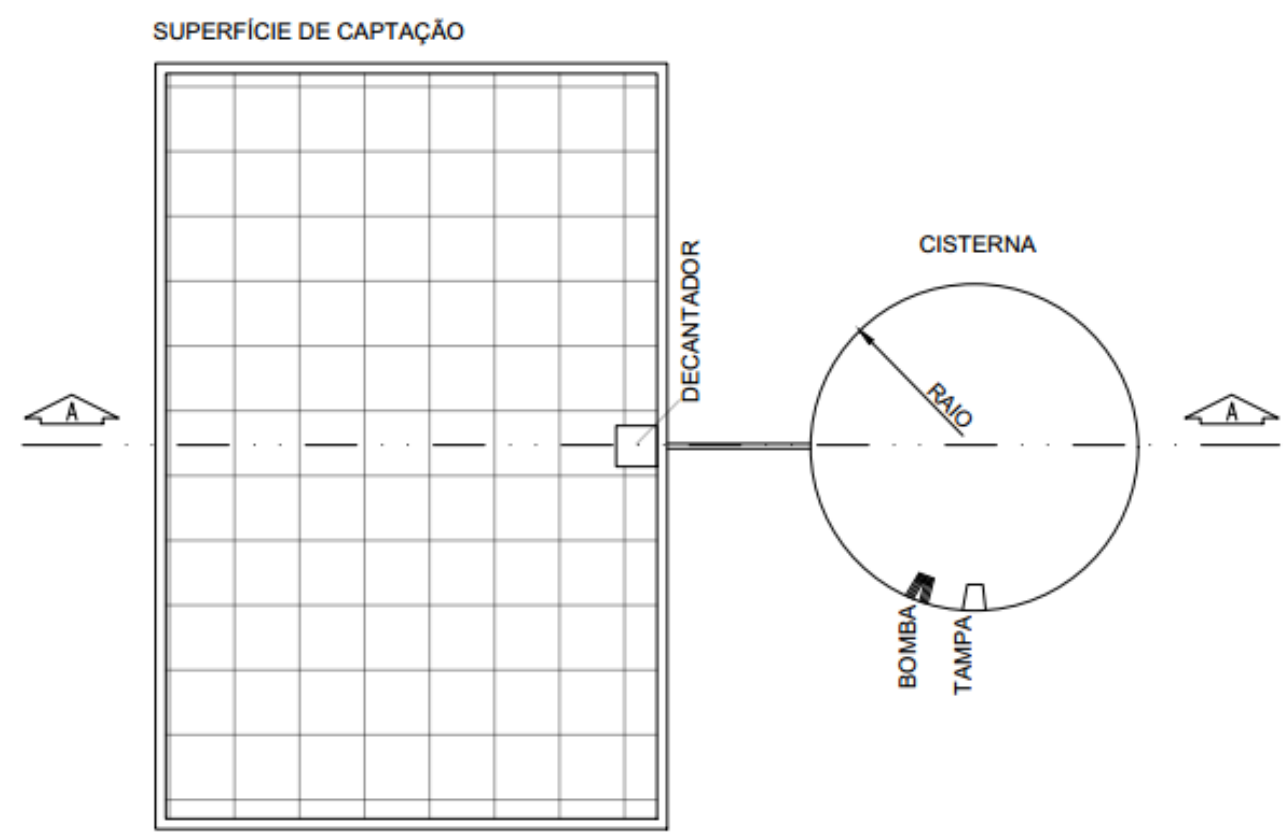

PLANTA BAIXA

Fonte: Os autores, 2019 
Os custos dos sistemas foram levantados em função do material e mão de obra necessários para a execução de cisternas de placas com diferentes volumes e áreas de captação do tipo calçadão, cuja superfície de captação é pavimentada com placas de concreto. Para a composição, utilizaram-se valores das bases de dados do Sistema Índices da Construção Civil (SINAPI, 2018) e do Sistema de Orçamento de Obras de Sergipe (ORSE, 2018), ambas atualizadas para o ano 2018. Foram feitas composições de custo para cisternas com volumes de 5 a $100 \mathrm{~m}^{3}$, possibilitando a interpolação de dados diferentes desse intervalo. Para a área de captação, foram levantados os custos para a execução de $1 \mathrm{~m}^{2}$ de calçadão, definido como o cenário mais econômico.

\section{RESULTADOS, ANÁLISE E DISCUSSÃO}

A Figura 3 mostra o comportamento do rendimento do sistema de aproveitamento de água de chuva para atendimento a uma demanda de $250 \mathrm{~L} /$ dia. Observa-se que o percentual de atendimento aumenta com o aumento do volume, tendendo a um valor máximo, limitado pela área de captação. A partir desse ponto, qualquer acréscimo do volume é inútil para o aumento do nível de atendimento. São avaliadas quatro situações representadas por diferentes valores de área de captação. A curva representante do sistema de $170 \mathrm{~m}^{2}$ de área de captação atinge o nível de atendimento de $95 \%$ apenas com um volume de 45 mil litros de reservatório, enquanto as curvas de área de captação de $200 \mathrm{~m}^{2}, 250 \mathrm{~m}^{2}$ e $300 \mathrm{~m}^{2}$ atingem o mesmo nível com volumes de reservatório de 22,5, 16,5 e 14,5 mil litros, respectivamente. Estas curvas demonstram que o nível de atendimento de $95 \%$ pode ser alcançado por várias combinações entre área de captação e volume de reservatório, o que constiui uma indeterminação matemática.

Visto de outra forma, a Figura 4 apresenta três curvas de potencial de atendimento em que são mostradas as diversas combinações entre volume de reservatório e área de captação para o atendimento da demanda de $250 \mathrm{~L} / \mathrm{d}$. As curvas tendem à estabilização no eixo das ordenadas, que representa a mínima área de captação possível para 0 atendimento aos níveis especificados, enquanto que a estabilização no eixo das abscissas representa o menor volume para o alcance dos níveis de atendimento. Isso quer dizer que, a partir de certo ponto, o aumento do volume ou área de captação é inútil para o alcance do nível de atendimento, contribuindo apenas para o aumento do custo do sistema.

Figura 3: Volume de reservatório x potencial de atendimento

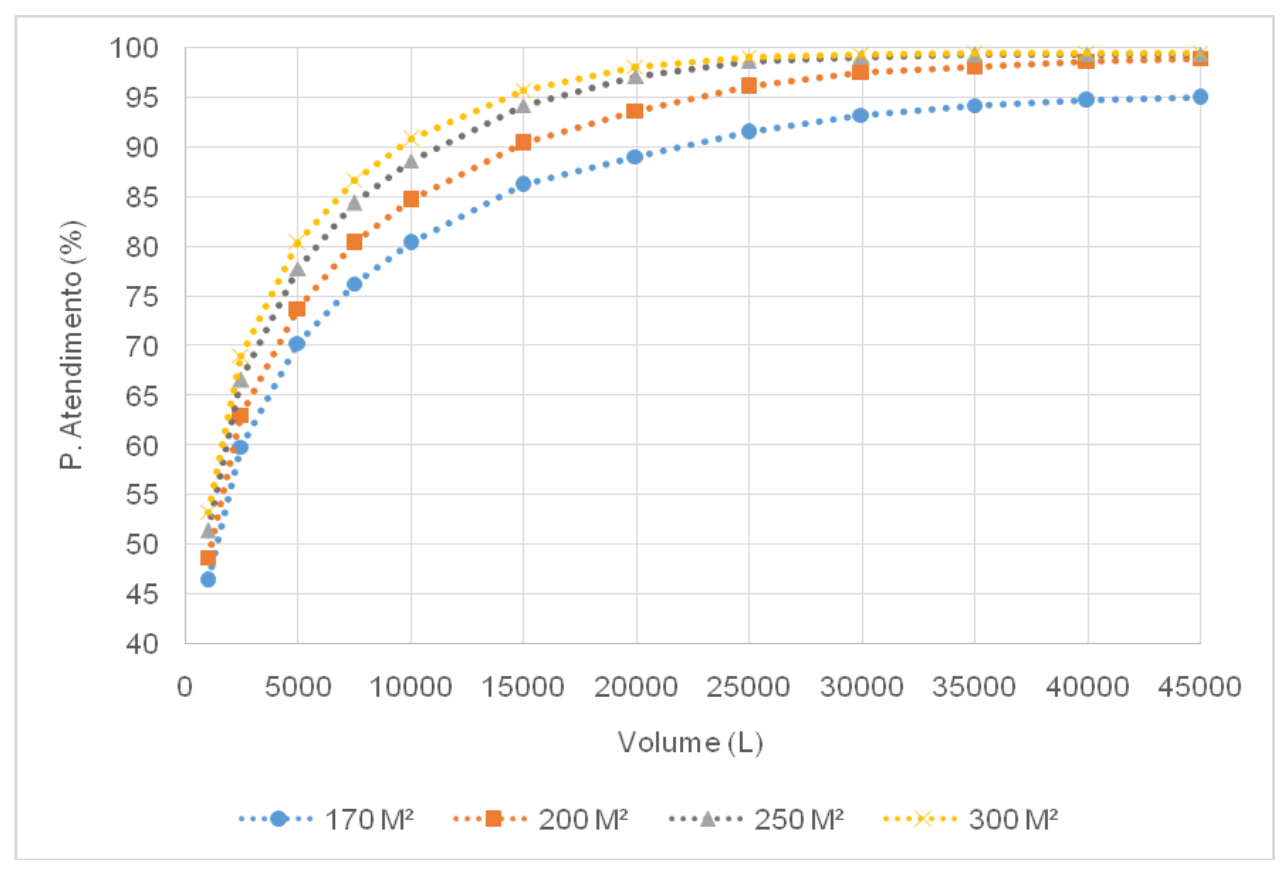

Fonte: Os autores, 2019. 
Figura 4: Combinação entre volumes de reservatório $x$ áreas de captação para níveis de atendimento específicos

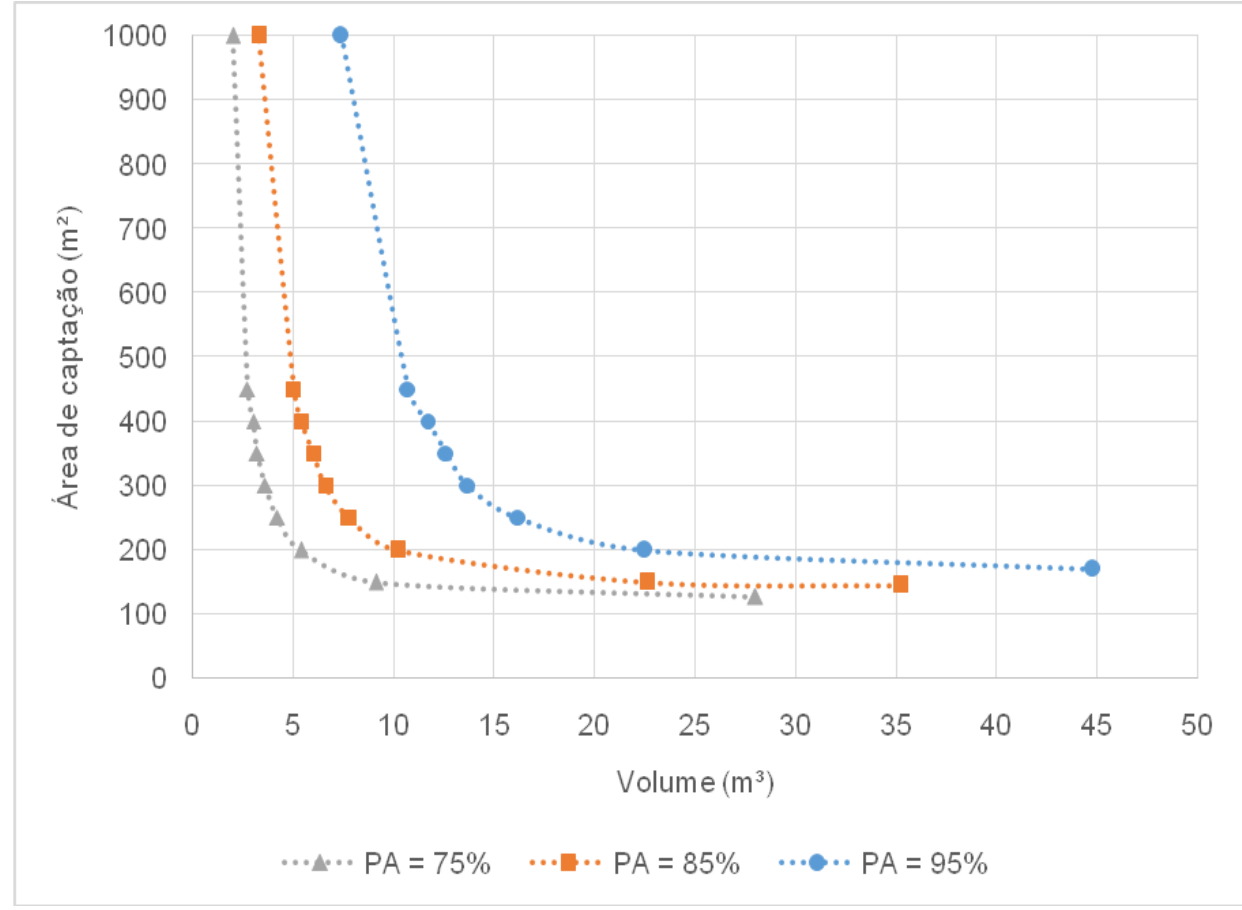

Fonte: Os autores, 2019.

O custo total dos sistemas foi obtido através de determinação das curvas de custo obtidas para cada um dos componentes do sistema de captação, conforme Figuras $5 a$ (cisterna) e $5 b$ (área de captação).

A curva da Figura $5 a$ é regida pela equação exponencial $y=1270 x V^{0,48}$ onde $\checkmark$ representa o volume do reservatório, em $\mathrm{m}^{3}$. O seu expoente é menor que 1 , o que significa que, com uma derivada que decresce com o aumento do volume do reservatório, os acréscimos no custo da execução são cada vez menores. Por outro lado, na Figura $5 b$, a equação de custo para execução da área de captação é regida por uma equação linear.

Com base na Tabela 1, o custo dos sistemas aumenta à medida em que 0 potencial de atendimento aumenta, e esta informação pode ser verificada se for adotado um volume de cisterna constante de $15 \mathrm{~m}^{3}$. Para a curva de potencial de atendimento de $75 \%$ (Figura 4), a área necessária para 0 atendimento, considerando o volume adotado, é de $132 \mathrm{~m}^{2}$, o que corresponde a um custo total do sistema de $R \$ 8.000,80$, enquanto para as curvas com potenciais de $85 \%$ e $95 \%$, as áreas necessárias são de $163 \mathrm{~m}^{2} \mathrm{e}$ $266 \mathrm{~m}^{2}$, respectivamente. Estes acréscimos representam um aumento do custo em relação ao primeiro sistema de $9,6 \%$ e $41,8 \%$, respectivamente.

Para um melhor entendimento do comportamento dos sistemas de captação, foram feitas simulações com potenciais de atendimento variando de $65 \%$ até $95 \%$, detalhados na Tabela 1. Para cada valor de atendimento à demanda, realizaram-se simulações para áreas de captação de 100 a $1000 \mathrm{~m}^{2}$. Com os valores de área, foram calculados os volumes necessários para 0 atendimento a cada demanda e, em seguida, 0 custo do sistema Área $\mathrm{x}$ Volume. Além disso, para cada atendimento à demanda, foram interpolados os menores custos de sistema e seus respectivos $F_{D}$, destacados em cinza na Tabela 1.

Para o esclarecimento da metodologia de obtenção dos sistemas ótimos, a Figura 6 relaciona as curvas de custo da cisterna, da área de captação e do sistema completo em função da área de captação, para um Potencial de atendimento de $95 \%$. 


\section{Figura 5: Custo para a construção de cisternas em função do volume (5a) e custo para construção de área de captação em função da área (5b)}

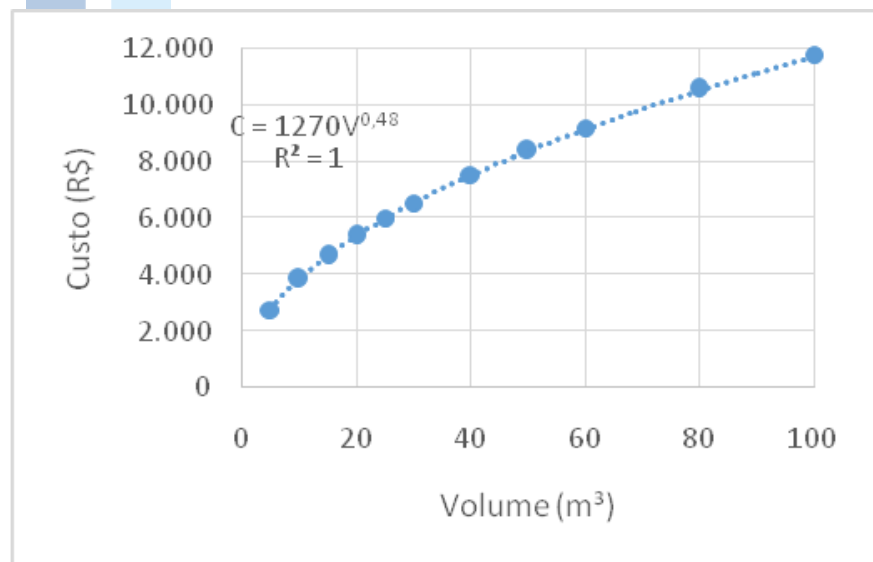

(a)

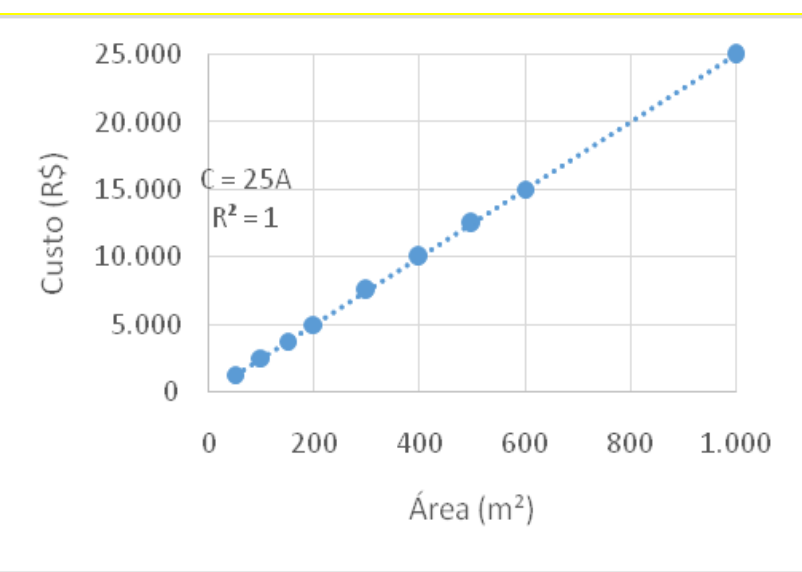

(b)

Fonte: Os autores, 2019.

De acordo com a análise das curvas de custo, pode ser verificado um ponto mínimo da curva de custo total, definida como o somatório das duas curvas anteriores. O volume do reservatório referente a esse ponto mínimo, juntamente à área correspondente, caracteriza a melhor opção de sistema para atender à demanda de 250L, com uma confiança de 95\% e menor custo econômico.

O parâmetro $F_{D}$ correspondente ao sistema ótimo, neste caso 0,66 , quando calculado para outras demandas, não apresentou variação. Este mesmo parâmetro, quando aplicado em regiões com precipitação média e confiança semelhantes às do caso estudado, constitui uma importante ferramenta para 0 dimensionamento ótimo de sistemas de captação de água da chuva, além de facilitar a gestão dos recursos hídricos nas regiões mais necessitadas e auxiliar na promoção da convivência com o semiárido.

\section{CONCLUSÃO}

O artigo evidencia a indeterminação matemática da combinação de área de captação e volume de cisterna para o atendimento de uma dada demanda. Os dados deixaram claro que o nível de atendimento apresenta um ponto de saturação, a partir do qual o aumento individual do volume da cisterna ou da área de captação não exerce praticamente nenhuma influência no aumento do potencial de atendimento, levando a um completo desperdício de recursos.

Foi, assim, apresentado um método que minimiza o custo total de sistemas de captação e armazenamento de água da chuva, o qual pode ser utilizado para qualquer regime pluviométrico. $O$ custo total do sistema cresce com o aumento do potencial de atendimento. Foram analisados potenciais de atendimento a partir de $65 \%$, o que pode ser útil na hipótese de se dispor de outras fontes confiáveis de água ou, em caso de restrição de recurso, para implantação de um sistema que atinja $95 \%$.

A aplicação do método para uma demanda de $250 \mathrm{~L} /$ dia e potencial de atendimento de $95 \%$ mostra que o sistema com área de captação de $208 \mathrm{~m}^{2}$ e volume de cisterna de $20,9 \mathrm{~m}^{3}$ oferece o menor custo entre os sistemas estudados, o que caracteriza a melhor opção. $O \quad F_{D}$ correspondente foi de 0,66 . 
Tabela 1: Características dos sistemas para vários potenciais de atendimento à demanda

\begin{tabular}{|c|c|c|c|c|c|c|c|c|}
\hline \multicolumn{9}{|c|}{ Potencial de atendimento: $65 \%$} \\
\hline Área / $\left(\mathrm{m}^{2}\right)$ & $\mathrm{F}_{\mathrm{D}}$ & Volume $/\left(\mathrm{m}^{3}\right)$ & \multicolumn{2}{|c|}{ Custo Área } & \multicolumn{2}{|c|}{ Custo Cisterna } & \multicolumn{2}{|c|}{ Custo total } \\
\hline 110 & 1,25 & 21 & $\mathrm{R} \$$ & $2.750,00$ & $\mathrm{R} \$$ & $5.524,90$ & $\mathrm{R} \$$ & $8.274,90$ \\
\hline 125 & 1,10 & 7,3 & $\mathrm{R} \$$ & $3.125,00$ & $\mathrm{RS}$ & $3.313,33$ & $\mathrm{R} \$$ & $6.438,33$ \\
\hline 141 & 0,98 & 5,1 & $\mathrm{R} \$$ & $3.521,25$ & $\mathrm{R} \$$ & $2.785,45$ & $\mathrm{R} \$$ & $6.306,70$ \\
\hline 150 & 0,92 & 4,4 & $\mathrm{R} \$$ & $3.750,00$ & $\mathrm{R} \$$ & $2.593,40$ & $\mathrm{R} \$$ & $6.343,40$ \\
\hline 200 & 0,69 & 2,8 & $\mathrm{R} \$$ & $5.000,00$ & $\mathrm{RS}$ & $2.083,92$ & $\mathrm{R} \$$ & $7.083,92$ \\
\hline 250 & 0,55 & 2,3 & $\mathrm{R} \$$ & $6.250,00$ & $\mathrm{R} \$$ & $1.894,71$ & $\mathrm{R} \$$ & $8.144,71$ \\
\hline 300 & 0,46 & 2 & $\mathrm{R} \$$ & $7.500,00$ & $\mathrm{R} \$$ & $1.770,80$ & $\mathrm{R} \$$ & $9.270,80$ \\
\hline 350 & 0,39 & 1,8 & $\mathrm{R} \$$ & $8.750,00$ & $\mathrm{RS}$ & $1.682,79$ & $\mathrm{R} \$$ & $10.432,79$ \\
\hline 400 & 0,34 & 1,7 & $\mathrm{R} \$$ & $10.000,00$ & $\mathrm{R} \$$ & $1.636,88$ & $\mathrm{R} \$$ & $11.636,88$ \\
\hline 450 & 0,31 & 1,6 & $\mathrm{R} \$$ & $11.250,00$ & $\mathrm{R} \$$ & $1.589,56$ & $\mathrm{R} \$$ & $12.839,56$ \\
\hline 1000 & 0,14 & 1,2 & $\mathrm{R} \$$ & $25.000,00$ & $\mathrm{R} \$$ & $1.382,99$ & $\mathrm{R} \$$ & $26.382,99$ \\
\hline \multicolumn{9}{|c|}{ Potencial de atendimento: $85 \%$} \\
\hline Área $/\left(\mathrm{m}^{2}\right)$ & $\mathrm{F}_{\mathrm{D}}$ & Volume / $\left(\mathrm{m}^{3}\right)$ & \multicolumn{2}{|c|}{ Custo Área } & \multicolumn{2}{|c|}{ Custo Cisterna } & \multicolumn{2}{|c|}{ Custo total } \\
\hline 145 & 0,95 & 35,2 & $\mathrm{R} \$$ & $3.625,00$ & $\mathrm{R} \$$ & $7.093,72$ & $\mathrm{R} \$$ & $10.718,72$ \\
\hline 150 & 0,92 & 22,6 & $\mathrm{R} \$$ & $3.750,00$ & $\mathrm{R} \$$ & $5.724,73$ & $\mathrm{R} \$$ & $9.474,73$ \\
\hline 173 & 0,80 & 13,1 & $\mathrm{R} \$$ & $4.319,75$ & $\mathrm{R} \$$ & $4.396,93$ & $\mathrm{R} \$$ & $8.716,68$ \\
\hline 200 & 0,69 & 10,2 & $\mathrm{R} \$$ & $5.000,00$ & $\mathrm{R} \$$ & $3.895,51$ & $\mathrm{R} \$$ & $8.895,51$ \\
\hline 250 & 0,55 & 7,8 & $\mathrm{R} \$$ & $6.250,00$ & $\mathrm{R} \$$ & $3.421,27$ & $\mathrm{R} \$$ & $9.671,27$ \\
\hline 300 & 0,46 & 6,6 & $\mathrm{R} \$$ & $7.500,00$ & $\mathrm{R} \$$ & $3.155,58$ & $\mathrm{R} \$$ & $10.655,58$ \\
\hline 350 & 0,39 & 6 & $\mathrm{R} \$$ & $8.750,00$ & $\mathrm{R} \$$ & $3.013,35$ & $\mathrm{R} \$$ & $11.763,35$ \\
\hline 400 & 0,34 & 5,4 & $\mathrm{R} \$$ & $10.000,00$ & $\mathrm{R} \$$ & $2.863,57$ & $\mathrm{R} \$$ & $12.863,57$ \\
\hline 450 & 0,31 & 5 & $\mathrm{R} \$$ & $11.250,00$ & $\mathrm{R} \$$ & $2.758,89$ & $\mathrm{R} \$$ & $14.008,89$ \\
\hline 1000 & 0,14 & 3,3 & $\mathrm{R} \$$ & $25.000,00$ & $\mathrm{R} \$$ & $2.256,37$ & $\mathrm{R} \$$ & $27.256,37$ \\
\hline
\end{tabular}

\begin{tabular}{|c|c|c|c|c|c|c|c|c|}
\hline \multirow{2}{*}{$\begin{array}{c}\text { Área / }\left(\mathrm{m}^{2}\right) \\
127\end{array}$} & \multirow{2}{*}{\begin{tabular}{|c|}
$F_{D}$ \\
1,08
\end{tabular}} & \multirow{2}{*}{$\frac{\text { Volume } /\left(\mathrm{m}^{3}\right)}{28}$} & \multicolumn{2}{|c|}{ Custo Área } & \multicolumn{2}{|c|}{ Custo Cisterna } & \multicolumn{2}{|c|}{ Custo total } \\
\hline & & & $\mathrm{RS}$ & $3.180,25$ & $\mathrm{R} \$$ & $6.350,12$ & $\mathrm{R} \$$ & $9.530,37$ \\
\hline 150 & 0,92 & 9,1 & $\mathrm{RS}$ & $3.750,00$ & $\mathrm{R} \$$ & $3.686,23$ & $\mathrm{R} \$$ & $7.436,23$ \\
\hline 158 & 0,87 & 8 & $\mathrm{RS}$ & $3.946,25$ & $\mathrm{R} \$$ & $3.463,44$ & $\mathrm{R} \$$ & $7.409,69$ \\
\hline 200 & 0,69 & 5,4 & $\mathrm{RS}$ & $5.000,00$ & $\mathrm{R} \$$ & $2.863,57$ & $\mathrm{R} \$$ & $7.863,57$ \\
\hline 250 & 0,55 & 4,2 & $\mathrm{RS}$ & $6.250,00$ & $\mathrm{R} \$$ & $2.535,67$ & $\mathrm{RS}$ & $8.785,67$ \\
\hline 300 & 0,46 & 3,6 & $\mathrm{R} \$$ & $7.500,00$ & $\mathrm{R} \$$ & $2.353,41$ & $\mathrm{R} \$$ & $9.853,41$ \\
\hline 350 & 0,39 & 3,2 & $\mathrm{R} \$$ & $8.750,00$ & $\mathrm{R} \$$ & $2.223,03$ & $\mathrm{R} \$$ & $10.973,03$ \\
\hline 400 & 0,34 & 3 & $\mathrm{R} \$$ & $10.000,00$ & $\mathrm{R} \$$ & $2.154,67$ & $\mathrm{R} \$$ & $12.154,67$ \\
\hline 450 & 0,31 & 2,7 & $\mathrm{R} \$$ & $11.250,00$ & $\mathrm{R} \$$ & $2.047,57$ & $\mathrm{R} \$$ & $13.297,57$ \\
\hline 1000 & 0,14 & 2 & $\mathrm{R} \$$ & $25.000,00$ & $\mathrm{R} \$$ & $1.770,80$ & $\mathrm{R} \$$ & $26.770,80$ \\
\hline
\end{tabular}

Fonte: Os autores, 2019.

Potencial de atendimento: $95 \%$
\begin{tabular}{ccccccccc}
\multicolumn{2}{l}{ Área $/\left(\mathrm{m}^{2}\right)$} & $\mathrm{F}_{\mathrm{D}}$ & Volume $/\left(\mathrm{m}^{3}\right)$ & Custo Área & Custo Cisterna & \multicolumn{2}{c}{ Custo total } \\
\hline 170 & 0,81 & 44,8 & $\mathrm{R} \$$ & $4.255,00$ & $\mathrm{R} \$$ & $7.971,79$ & $\mathrm{R} \$$ & $12.226,79$ \\
200 & 0,69 & 22,5 & $\mathrm{R} \$$ & $5.000,00$ & $\mathrm{R} \$$ & $5.712,46$ & $\mathrm{R} \$$ & $10.712,46$ \\
208 & 0,66 & 20,9 & $\mathrm{R} \$$ & $5.196,25$ & $\mathrm{R} \$$ & $5.512,15$ & $\mathrm{R} \$$ & $10.708,40$ \\
250 & 0,55 & 16,2 & $\mathrm{R} \$$ & $6.250,00$ & $\mathrm{R} \$$ & $4.872,89$ & $\mathrm{R} \$$ & $11.122,89$ \\
300 & 0,46 & 13,7 & $\mathrm{R} \$$ & $7.500,00$ & $\mathrm{R} \$$ & $4.493,26$ & $\mathrm{R} \$$ & $11.993,26$ \\
350 & 0,39 & 12,6 & $\mathrm{R} \$$ & $8.750,00$ & $\mathrm{R} \$$ & $4.314,91$ & $\mathrm{R} \$$ & $13.064,91$ \\
400 & 0,34 & 11,7 & $\mathrm{R} \$$ & $10.000,00$ & $\mathrm{R} \$$ & $4.162,91$ & $\mathrm{R} \$$ & $14.162,91$ \\
450 & 0,31 & 10,7 & $\mathrm{R} \$$ & $11.250,00$ & $\mathrm{R} \$$ & $3.986,77$ & $\mathrm{R} \$$ & $15.236,77$ \\
1000 & 0,14 & 7,4 & $\mathrm{R} \$$ & $25.000,00$ & $\mathrm{R} \$$ & $3.335,21$ & $\mathrm{R} \$$ & $28.335,21$ \\
\hline
\end{tabular}

Figura 6: Curva de custos em função da área de captação para um potencial de atendimento de $95 \%$

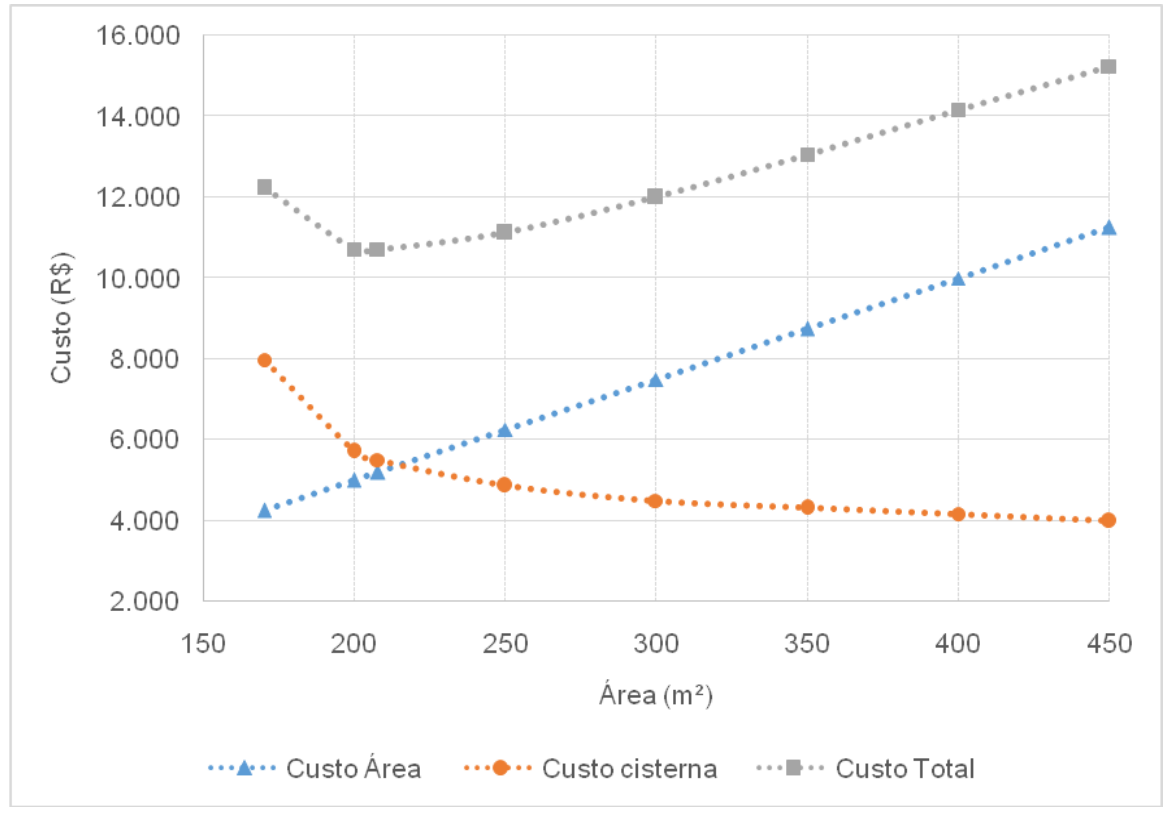

Fonte: Os autores, 2019.

\section{REFERÊNCIAS}

AZEVÊDO, E. L.; ALVES, R. R. N.; DIAS, T. L. P.; MOLOZZI, J. How do people gain access to water resources in the Brazilian semiarid
(Caatinga) in times of climate change? Environmental Monitoring And Assessment, [s.I.], v. 189, n. 8, p.1-17, 5 jul. 2017. Springer Nature. 
CAMPISANO, A.; BUTLER, D.; WARD, S.; BURNS, M. J.; FRIEDLER, E.; DEBUSK, K.; FISHER-JEFFES, L. N.; GHISI, E.; RAHMAN, A.; FURUMAI, H.; HAN, M. Urban rainwater harvesting systems: research, implementation and future perspectives. Water Research, SI, v. 115, n. 1, p.195-209, mar. 2017.

MODICA, C. Regional scale analysis for the design of storage tanks for domestic rainwater harvesting systems. Water Science And Technology, [s.l.], v. 66, n. 1, p.1-8, jul. 2012.

CIRILO, J. A. Políticas públicas de recursos hídricos para o semiárido. Estudos Avançados, v.22, p. 61-82, 2008.

ELLIOTT, J.; DERYNG, D.; MÜLLER, C.; FRIELER, K.; KONZMANN, M.; GERTEN, D.; GLOTTER, M. FLÖRKE, M.; WADA, Y.; BEST, N.; EISNER, S.; FAKETE, B. M.; FOLBERTH, C.; FOSTER, I.; GOSLING, S. N.; HADDELAND, I.; KHABAROV, N.; LUDWIG, F.; MASAKI, Y.; OLIN, S.; ROSENZWEIG, C.; RUANE, A. C.; SATOH, Y.; SCHMID, E.; STACKE, T.; TANG, Q.; WISSER, D. Constraints and potentials of future irrigation water availability on agricultural production under climate change. PNAS, Chicago, v. 111, n. 9, p. 3239-3244, mar. 2014.

FEWKES, A. Modelling the performance of rainwater collection systems: towards a generalised approach. Urban Water 1, Nottingham, v. 1, n. 4, p. 323-333, dez. 2000.

FRANÇA, F. M. C.; HOLANDA JUNIOR; E. V.; SOUSA NETO, J. M. de $S$. Análise da viabilidade financeira e econômica do modelo de exploração de ovinos e caprinos no Ceará por meio do sistema agrossilvipastoril. Documentos Técnicos Científicos, [s. I.], v. 42, n. 2, p. 287-308, 2011.

GUALDANI, C.; FERNÁNDEZ, L.; GUILLÉN, M. L. Convivência com o semiárido brasileiro: reaplicando saberes através das tecnologias sociais. Brasília: Labs, 2015. 172 p.

IBGE - INSTITUTO BRASILEIRO DE GEOgRAFIA E ESTAtísticA. Pesquisa de produção pecuária. 2016. Rio de Janeiro: IBGE.

INMET - Instituto Nacional de Meteorologia. Disponível em:<http://www.inmet.gov.br>. Acesso em: 22 fev.2018.
JENKINS, D.; PEARSON, F. Feasibility of rainwater collection systems in California. Water Resources Center, California, v. 4, n. 1, p.1-65, ago. 1978.

LATHAM, B. G. Rainwater collection systems: the design of single-purpose reservoirs. National Library, Canada, v. 6, n. 2, p.1-257, 1983.

KALIBER, M.; KOLUMAN, N.; SILANIKOVE, N. Physiological and behavioral basis for the successful adaptation of goats to severe water restriction under hot environmental conditions. [s. I.], n. 2016, p. 82-88, 2017.

LONDRA, P. A.; THEOCHARIS, A. T.; BALTAS, E.; TSIHRINTZIS, V. A. Optimal sizing of rainwater harvesting tanks for domestic use in Greece. Water Resources Management, v. 29, n. 12, p. 4357-4377, jul. 2015.

MAIA, A. G.; CESANO, D.; MIYAMOTO, C. B.; EUSEBIO, G. S.; SILVA, P. A. de O. Climate change and farm-level adaptation: the Brazilian Sertão. [s. I.], v. 10, n. 5, p. 729-751, 2018.

MONTENEGRO, A. A. A.; MONTENEGRO, S. M. G. L. Olhares sobre as políticas públicas de recursos hídricos para o semiárido. In: GHEYI, H. R.; PAZ, V. P. da S.; MEDEIROS, S. de S.; GALVÃO, C. de O. Recursos Hídricos Regiões Semiáridas: estudos e aplicações. Campina Grande: INSA, 2012. p. 2-24.

MORAES NETO, O.T.; RODRIGUES, A.; ALBUQUERQUE, A.C.A.; MAYER. S. Manual de capacitação de agentes de desenvolvimento rural (ADRs) para a Caprinovino cultura. João Pessoa-PB: SEBRAE/PB, 2003. 114 p.

ORSE - Sistema de Orçamento de Obras de Sergipe. Disponível em: $<$ https://www.cehop.se.gov.br/orse/>. Acesso em: 23 mai. 2018.

PALHARES, J. C. P.; GUIDONI, A. L.. Qualidade da água de chuva armazenada em cisterna utilizada na dessedentação de suínos e bovinos de corte. Ambiente \& Água, [s. I.], v. 7 , n. 1, p. 244-254, 2012.

SINAPI - Índices da Construção Civil. Disponível em:<http://goo.gl/ttgltv>. Acesso em: 23 mai. 2018. 